
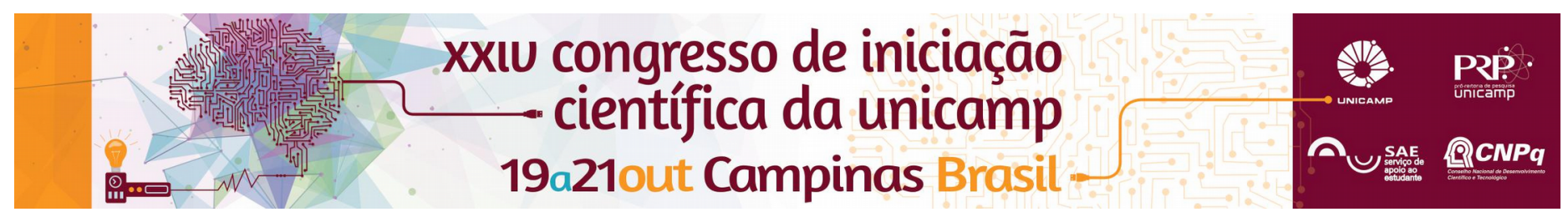

\title{
Simetrias e leis de conservação
}

\author{
Rafael L. Raiser*, Prof. Dra. Arlene C. Aguilar
}

\section{Resumo}

Neste trabalho, vamos apresentar como as simetrias físicas presentes em sistemas clássicos e quânticos estão relacionadas diretamente às leis de conservação através do Teorema de Noether.

\section{Palavras-chave:}

Simetrias, leis de conservação, Teorema de Noether

\section{Introdução}

Ao estudar física, nos deparamos com uma série de leis, como por exemplo a primeira lei de movimento de Newton sobre a conservação do momento ou a primeira lei da termodinâmica, sobre a conservação de energia. Neste trabalho, vamos discutir como algumas dessas leis de conservação surgem devido a propriedades de simetria do sistema em questão.

Esta conexão entre simetrias e leis de conservação foi formulada em 1918 através do Teorema de Noether ${ }^{1}$, que se tornou um dos principais pilares da física moderna.

\section{Resultados e Discussão}

Em física, dizemos que um sistema é simétrico se as equações que descrevem sua dinâmica são invariantes sob determinada operação. $\mathrm{Na}$ mecânica clássica, sabemos que os resultados físicos obtidos devem ser sempre invariantes por (i) translação espacial, (ii) translação temporal e (iii) rotação.

Com o auxílio das ferramentas das mecânicas Lagrangiana e Hamiltoniana², é possível derivar as leis de conservação associadas às simetrias acima. O procedimento é simples: escrevemos o Lagrangiano,

$L$, ou Hamiltoniano, $H$, do sistema, e verificamos que ao aplicarmos transformações do tipo

$$
\begin{gathered}
\text { (i) } q_{i} \rightarrow q_{i}{ }^{\prime}=q_{i}+\varepsilon_{i} ; \\
(i i) t \rightarrow t+\varepsilon ; \\
\text { (iii) } x \rightarrow x-\varepsilon y, y \rightarrow y+\varepsilon x,
\end{gathered}
$$

é possível mostrar que a variação de $L$ e $H$ devido à transformação será nula, isto é,

$$
\delta L=\delta H=0 \text {, }
$$

se e somente se as leis de conservação mostradas na Tabela 1 forem obedecidas.

Esta conexão também pode ser provada em nível quântico. Um exemplo simples é uma translação espacial infinitesimal de $\varepsilon$ na coordenada $x$ da função de onda $\psi(x)$, de forma que

$$
\psi(x) \rightarrow \psi(x-\varepsilon)=\psi(x)-\varepsilon \frac{d \psi}{d x}+O\left(\varepsilon^{2}\right) .
$$

Ao aplicamos esta mudança na definição do valor esperado de $\boldsymbol{L}$, verificamos que esta quantidade será invariante se o momento quântico

$p_{x}$ for conservado.

Também podemos abordar as simetrias quânticas contínuas, interpretadas como um conjunto de transformações infinitesimais. Uma lei de conservação que pode ser derivada dentro deste contexto é a conservação do isospin de uma sistema de partículas elementares.

Tabela 1. Simetria e lei de conservação associada em sistemas clássicos.

\begin{tabular}{lc}
\hline \multicolumn{1}{c}{ Simetria } & Quantidade conservada \\
\hline i) Translação espacial & Momento \\
\hline ii) Translação temporal & Energia \\
\hline iii) Rotação & Momento angular \\
\hline
\end{tabular}

Por fim, estudando as chamadas simetrias locais $^{3}$ - simetrias cujos parâmetros dependem das coordenadas do espaço-tempo, é possível mostrar que a Equação de Schrödinger é invariante por uma transformação de fase na função de onda do tipo

$$
\psi(\vec{r}) \rightarrow \exp (i \alpha \vec{r}) \psi(\vec{r})
$$

se o operador gradiente e o potencial vetor $\vec{A}$ forem simultaneamente modificados da seguinte forma:

$$
\nabla \rightarrow \nabla-i \vec{A}(\vec{r}), \operatorname{com} \vec{A}(\vec{r}) \rightarrow \vec{A}(\vec{r})+\nabla \alpha(\vec{r}) \text {. }
$$

Esta modificação é chamada de transformação de gauge, e nos diz que a invariância sob uma transformação de mudança de fase requer a introdução de campos adicionais, denominados de campos de gauge.

\section{Conclusão}

Este trabalho permitiu uma maior compreensão sobre a origem das leis de conservação, tanto em sistemas clássicos quanto quânticos. Derivamos as leis de conservação de energia, momento e momento angular para sistemas clássicos. O estudo de simetrias locais nos levou a uma breve introdução à necessidade de transformações de gauge para manter a equação de Schrödinger invariante sob mudança de fase.

\section{Agradecimentos}

Ao CNPq, pelo apoio financeiro para este projeto, através do programa PIBIC.

'Noether E. (1918). Invariante Variationsprobleme. Nachr. D. König.

2TAYLOR, John R., Classical mechanics, Sausalito, Calif.: Univ. Science, c2005. 786 p., il. ISBN 189138922X (enc.).

${ }^{3}$ FERBEL, Thomas (Coaut. de); DAS, Ashok. Introduction to nuclear and particle physics. New York, NY: John Wiley, c1994. xvi, 327p., il. 